\title{
Analysis of ten years of social oocyte cryopreservation: a research article
}

\author{
Lorraine Kasaven ${ }^{1}$, Benjamin Jones $^{2}$, Carleen Heath ${ }^{3}$, Rabi Odia ${ }^{3}$, Joy Green ${ }^{3}$, Aviva \\ Petrie $^{4}$, Srdjan Saso ${ }^{2}$, Paul Serhal ${ }^{3}$, and Jara Ben-Nagi ${ }^{5}$ \\ ${ }^{1}$ Imperial College Healthcare NHS Trust \\ ${ }^{2}$ Imperial College London \\ ${ }^{3}$ Centre for Reproductive and Genetic Health \\ ${ }^{4}$ University College London \\ ${ }^{5}$ The Centre for Reproductive and Genetic Health (CRGH)
}

October 9, 2020

\begin{abstract}
Objective: To assess the relationship between number of oocytes retrieved during social egg freezing (SEF) cycles with various clinical, biochemical and radiological markers; e.g. age, body mass index (BMI), baseline anti-Mullerian hormone (AMH), antral follicle count (AFC), Oestradiol level (E2) and total number of follicles [?]12mm at trigger. Main outcome measures: To describe the characteristics and outcomes of women who underwent SEF. Methods: A retrospective cohort of women embarking on SEF between 2008 and 2018 from a single London UK fertility clinic. Results: 483 stimulation cycles were undertaken in 373 women. The median age at freeze was 38 (26-47) years. The median numbers of oocytes retrieved per cycle was 8 (037 , and total oocytes cryopreserved 8 (0-45) per woman. BMI, E2 level and number of follicles [?]12mm at trigger were all significant predictors of oocyte yield. Multivariate analysis confirmed no significant relationship between AFC or AMH, whilst on univariate analysis statistical significance was proven. 36 women returned to use their oocytes, with 41 autologous egg thaw cycles undertaken. 12 successful livebirths were achieved by 11 women. The overall livebirth rate was $26.8 \%$ per cycle. No livebirths occurred in women [?]40 years old and $82 \%$ of all livebirths were in women aged 36-39 at freeze. Conclusions: This study demonstrates clinical, biochemical and radiological markers can predict oocyte yield in SEF cycles. However, subsequent reproductive outcomes highlight women embarking upon SEF should be encouraged to do so before the age of 37 years, and no later than 40 to optimise successful livebirth.
\end{abstract}

\section{Introduction}

The development of oocyte vitrification, with success rates now similar to fresh cycles, ${ }^{1}$ has enabled the opportunity to electively cryopreserve oocytes prior to the physiological decline in oocyte quality and quantity. Referred to herein as social egg freezing (SEF), it negates the age-associated deterioration in reproductive potential, but remains a controversial option owing to the multitude of ethical, legal, economic and obstetric related issues that it provokes. ${ }^{2}$ Whilst some have little alternative option, such as single women approaching their late-thirties who desire biologically related children, ${ }^{3}$ other indications include delaying childbearing for furthering education or to focus on career. ${ }^{4}$

Despite the theoretical benefit of preserving reproductive potential through SEF, it does not guarantee against involuntary childlessness. ${ }^{5}$ Following SEF, if women return to use their cryopreserved oocytes; risk of loss during thawing or following unsuccessful embryo transfer may result in potential exhaustion of cryopreserved oocytes prior to achieving livebirth. Replenishment may subsequently be difficult in the same woman at a later age owing to the inevitable physiological depletion in ovarian reserve. ${ }^{6}$ Therefore, at the 
time of SEF, it is important to maximise the number of oocytes retrieved for cryopreservation to improve the probability of a successful livebirth. ${ }^{7}$

Various characteristics are associated with oocyte yield such as age at time of oocyte retrieval. ${ }^{8}$ This encapsulates the very principle of SEF, and directly relates to the progressive depletion in primordial follicles and reduced neuroendocrine response to ovarian stimulation. This clinically manifests with poorer oocyte quantity and quality, as demonstrated by multivariate analysis to control for other confounding variables. ${ }^{9}$ Contrarily, increased BMI is associated with reduction in yield. ${ }^{10}$

The most accurate markers of ovarian reserve considered for prediction of oocyte yield following controlled ovarian stimulation are antral follicle count (AFC) and anti-Mullerian hormone (AMH) ${ }^{11}$ Endocrine markers including Oestradiol (E2), a measure of granulosa cell function has also been used. ${ }^{12}$ Elevated levels of follicle stimulating hormone (FSH) on day 2-3 of a cycle is associated with a significant reduction in yield, when compared to women with lower FSH of the same age. ${ }^{13}$ Radiologically, a follicular volume between 13$23 \mathrm{~mm} / 1-6 \mathrm{~mL}$ at the time of ovulation trigger is also associated with optimal oocyte yields. ${ }^{14}$

The aim of this study was to assess the relationship between oocyte yield with clinical, biochemical and radiological markers including age, BMI, $\mathrm{AMH}, \mathrm{AFC}, \mathrm{E} 2$ and total number of follicles [?]12 $\mathrm{mm}$ at trigger. A secondary aim was to report the reproductive outcomes following SEF in one of the largest reported cohorts in the UK so far.

\section{Methods}

\section{Selection criteria}

This retrospective cohort includes all women who approached the Centre for Reproductive and Genetic Health in London, UK for SEF over a ten-year period between 2008 and 2018.

\section{Clinical Protocols}

An initial assessment was carried out, which included a blood test for AMH (Roche Assay), FSH, E2 and ultrasound for AFC performed between days 1-4 of the menstrual cycle. The ovarian stimulation protocol was individualised depending on age, AFC, AMH and BMI. Ovarian stimulation using an antagonist protocol was commenced on the third day of the menstrual cycle. From day 6 onwards, the gonadotrophin dose was adjusted according to E2 levels and ultrasound evidence of stimulation. On day 7 of the cycle and/or once the leading follicle was [?]14 mm in diameter, Cetrorelix (Cetrotide) $0.25 \mathrm{mg}$ (Merck Serono, Germany) was commenced. The choice of trigger was individualised after assessment of age, baseline AFC and AMH, number of follicles and E2 levels on the day of trigger. Women considered low responders were given either Pregnynl a human chorionic gonadotrophin (hCG) trigger, or dual trigger with Pregnyl 1,500 IU (Organon, Netherlands) and Suprefact $1 \mathrm{ml}$ (Sanofi-Aventis, Germany), a gonadotrophin releasing hormone analogue $(\mathrm{GnRH})$. Women considered normal or high responders were given Suprefact only. Oocyte maturation was triggered once the mean diameter of [?]3 follicles [?]18mm. In the event women were at high risk of ovarian hyperstimulation syndrome (OHSS), Suprefact $1 \mathrm{ml}$ (Sanofi-Aventis, Germany) was administered and LH level was measured 8-12 hours later. Oocyte retrieval was scheduled 37 hours after ovulation trigger. Denudation was carried out 39 hours post the trigger injection and metaphase II oocytes were vitrified immediately after.

All thaw cycles included within our study refer to the thawing of oocytes which were frozen initially at $\mathrm{CRGH}$, undergoing the vitrification method. Vitrification at room temperature $\left(24-26^{\circ} \mathrm{C}\right)$ took place in two steps. Firstly, once oocytes were denuded they were moved for 12-15 minutes within a solution containing 7.5\% Dimethyl Sulfoxide-D6 (DMSO) and 7.5\% Ethylene Glycerol (EG). Oocytes achieving full recovery to normal size were then transferred to a vitrification solution consisting of $15 \%$ DMSO and EG plus 0.5-m sucrose for 60 seconds. Secondly, oocytes were then transferred via Cryotop straws at fast rate into liquid nitrogen for storage, within a minimum volume of vitrification solution. Six welled dishes adjacent to three welled plates from Cryotech were used containing solutions warmed at $37^{\circ}$ with various concentrations of sucrose (1.0. 0.5 and $0.0 \mathrm{~m})$. The vitrified oocytes within the Cryotop straws were then removed from storage and moved to liquid nitrogen, within $0.7 \mathrm{ml} 1.0 \mathrm{M}$ of sucrose, followed by $3-5$ minutes within $50 \mu$ l of varying 
decreasing sucrose concentrations at room temperature. Oocytes with full recovery were then transferred for 2 hours into culture before undergoing intracytoplasmic sperm injection (ICSI).

The oocytes were assessed 16-18 $\mathrm{h}$ post ICSI for the presence of pronuclei and were separated according to the number of pronuclei present. Oocytes displaying abnormal pronuclei numbers (zero, one and more than three) were either discarded or kept in a separate dish of cleavage medium (Vitrolife). Oocytes displaying 2 pronuclei were either moved to a fresh dish of cleavage medium (Vitrolife) while the two-step culture system was in effect, or single-step culture media (Sage/Origio). The embryos were either moved to Blastocyst medium (Vitrolife) or a fresh dish of single-step media (Sage/Origio) on day 3 in order to be cultured to the blastocyst stage, until day 5 or 6 post insemination. Any embryos that successfully formed a blastocyst on day 5 or 6 of culture (exhibiting the presence of clear inner cell mass and trophectoderm cell lines) were selected for vitrification.

Blastocyst vitrification took place at $37^{\circ} \mathrm{C}$. Multiple 5 -well Nunc dishes were used to warm $1 \times 0.5 \mathrm{ml}$ wells of V1 per blastocyst, $1 \times 0.5 \mathrm{ml}$ well of V2 and $1 \times 1 \mathrm{ml}$ well of V3 media solutions (Blastocyst Vitrification Kit, Sydney IVF) to $37^{\circ} \mathrm{C}$ a minimum of 1 hour prior to use. Blastocysts were isolated into micro-drops of warm Hepes media under oil, assessed at 200x magnification and graded as per the CRGH in-house grading criteria (adapted Cornell). Blastocoelic cavity collapse was then initiated by a single laser shot to the junction of two outermost trophectodermal cells, before the blastocysts were transferred to individual wells of the warmed V1 media (up to 4 blastocysts per dish). Vitrification was conducted by moving a single blastocyst from V1 to V2 media for 2 minutes followed by thorough washing through 3 drops of V3 media for 30-35 seconds. The blastocyst was then loaded rapidly onto a Cryolock with minimal volume of vitrification solution and plunged into liquid nitrogen before placing a protective cap over the loading strip. Once blastocysts were frozen, women embarked on a single elective embryo transfer. A medicated frozen embryo transfer was performed. ${ }^{15}$ All patients were advised to perform a urinary pregnancy test 16 days later.

Details of Ethics Approval

Ethics approval was not necessary for this retrospective study as vitrification of oocytes is a validated technique that should no longer be considered experimental.

\section{Statistical analyses}

SPSS version 24 software (SPSS, Chicago, Illinois, USA) was used for analysis. Descriptive statistical analysis was described as median \pm range. Multivariate Poisson regression was used to evaluate the effect of the aforementioned variables with the number of oocytes retrieved. Mann Whitney U test was used to determine a relationship between the variables and their effect on OHSS. The Kruskal Wallis test and Mann Whitney $\mathrm{U}$ tests were used to determine significance between age groups and probability of livebirth based upon their age and number of oocytes retrieved. Chi squared testing was utilised to determine statistical significance in those who returned to use their oocytes, to assess between age groups and achievement of livebirth. Statistical significance was set at $\mathrm{p}<0.05$.

\section{Results}

483 stimulation cycles were undertaken in 373 women. The median age (SD) at freeze was $38.3( \pm 3.5)$. The median number of cycles per woman was 1 (range 1-8). The median number of oocytes retrieved per cycle was 8 (0-37), whilst the number of metaphase II oocytes was 6 (0-28). The median BMI was 22.5 (17.1-37) $\mathrm{kg} / \mathrm{m}$. The median baseline AMH was $9.9(0.17-56.6) \mathrm{pmol} / \mathrm{l}$ and the AFC was 12 (2-49). At ovulation trigger, the median E2 level was $7.01(0.25-30.26)$ per $1000 \mathrm{pmol} / \mathrm{l}$ and the total number of follicles [?]12mm was 9 (1-50). (Table 1)

Univariable Poisson regression was used to assess the effect of each variable on oocyte yield (Table 2) . This identified that age, baseline AFC, AMH, E2, and follicle count [?]12mm on the day of trigger were all significant predictors of oocyte yield $(\mathrm{p}<0.001)$. Multivariable Poisson regression subsequently demonstrated that BMI (Incidence rate ratio; IRR; 1.02; 95\% CI $1.00-1.03 ; 95 \%$ p=0.04), E2 level per 1000 pmol/l increase (IRR 1.05; 95\% CI $1.04-1.07 ; \mathrm{p}<0.001$ ) and the number of follicles [?]12mm at trigger (IRR 1.02; 95\% CI 
1.01-1.03; $\mathrm{p}<0.001$ ) were all significant predictors of oocyte yield (Table 3) . However, there was no significant relationship between AFC (IRR=1.00; 95\% CI 0.99-1.02 $\mathrm{p}=0.43$ ) or AMH (IRR 1.00; 95\% CI $0.99-1.01 ; \mathrm{p}=0.71)$ with oocyte yield. An increase in age by one-year resulted in a $4 \%$ reduction in yield after adjusting for all other variables (IRR 0.96; 95\% CI 0.94-0.98; $\mathrm{p}<0.001$ ).

There were nine cases in total (2.4\%) of OHSS; all of which classified as mild in severity as per the Royal college of Obstetrician and Gynaecologist classification. ${ }^{16}$ In regards to mode of trigger, there were four cases of hCG, four Suprefact and one case of dual trigger. There were no associated hospital admissions or significant adverse events. Analysis using the Mann Whitney U test identified that age, total AFC, number of follicles more than $12 \mathrm{~mm}$ on the day of trigger and FSH were all significant predictors of OHSS $(\mathrm{p}<0.05)$. BMI, AMH and E2 levels however were not significant predictors of OHSS (Table 4).

Thirty-six women subsequently underwent 41 oocyte thaw cycles. The mean age at thaw was $42(+-4.1)$. The mean number of years between cryopreservation and thaw was 3.7 years. Almost two thirds $(\mathrm{n}=23)$ of women used partners sperm, whereas $36 \%(\mathrm{n}=13)$ used donor sperm. Of those who used donor sperm, the majority did so because they were single $(\mathrm{n}=9,69 \%)$.

The percentage of oocytes which survived thaw was $81,76 \%, 67.5 \%$ in women aged [?] 35, 36-39 and [?]40 years respectively. The fertilization rate of the frozen thawed oocyte was $53 \%, 68 \%$ and $58 \%$ in the respective age groups. There were 37 embryo transfers (ET). Almost half of the embryo transfers took place at the blastocyst stage $48.6 \%,(\mathrm{n}=18)$. Of those cycles with all embryos cultured to the blastocyst stage, the average number of blastocysts formed were $55.6 \%(10 / 18), 45.5 \%(40 / 88)$ and $50 \%(9 / 18)$ in the respective age groups.

Following embryo transfer, 13 women had confirmed clinical pregnancies resulting in a clinical pregnancy rate (fetal heart visualised on ultrasound) of $31.7 \%$ per thaw cycle. 12 livebirths were subsequently achieved by 11 women (one twins), attributing a livebirth rate of $26.8 \%$ (11/41) per cycle and $29.7 \%$ per ET (11/37). The remaining two women had missed miscarriages, having both first cryopreserved their oocytes above 40 years old.

In women aged [?]35 years, two livebirths from six egg thaw cycles were achieved, resulting in a livebirth rate per egg thaw cycle of $33 \%$. In those aged 36-39, 24 cycles led to 9 livebirths resulting in a livebirth rate of $37.5 \%$ per egg thaw cycle. In those aged 40 years and over, all 11 cycles were unsuccessful, resulting in a livebirth rate of $0 \%$ as demonstrated in Figure 1. The average age at cryopreservation of those who achieved a livebirth was 36.4, whereas those who did not was $39.0(\mathrm{p}=0.07)$.

\section{Discussion}

\section{Interpretation}

This study demonstrates herein that markers such as E2, BMI and the number of follicles [?]12mm at trigger are all independent significant predictors of oocyte yield in women undergoing SEF. However, whilst AFC and AMH were significant predictors of oocyte yield utilising univariate analysis, the relationship was not significant when controlling for other potential confounders. Whilst there are few previous studies assessing these relationships in the context of SEF, comparisons can be made from data from cycles performed for other indications, with consideration for the differences in population characteristics.

AMH is considered a reliable predictor of oocyte yield. ${ }^{17}$ However, it is well known that AMH levels are strongly correlated with age. ${ }^{18}$ This study demonstrates herein that AMH was a statistically significant predictor of yield utilising univariate analysis, but when controlling for other variables using multivariate analysis, no significant relationship was identified. We propose therefore, that when assessing oocyte yield controlling for confounders such as age; such interrelationships may nullify the impact of AMH.

It is important to emphasise that women undergoing SEF are young and fertile, unlike the vast majority of women undergoing IVF. AMH is often used as a surrogate marker of ovarian reserve in women undergoing ART, and therefore much of the evidence is limited to women with diminished ovarian reserve. ${ }^{19}$ Causes 
of subfertility include previous ovarian surgery or following gonadotoxic treatment with chemotherapy or radiotherapy, resulting in diminished AMH regardless of age ${ }^{20}$ As such it is unsurprising that data in infertile populations; AMH has been shown to be an independent significant predictor of oocyte yield. ${ }^{21}$ However, as demonstrated herein, in women who undergo SEF, age remained an independent significant predictor even when controlling for other variables, highlighting it as a more accurate predictor of oocyte yield than AMH in this population.

BMI is also associated with oocyte yield. ${ }^{22}$ However, whereas it was previously identified that oocyte quantity was lower in obese women, compared with those with a normal BMI ${ }^{23}$ the findings demonstrated herein contrarily show that oocyte yield increased by $2 \% \mathrm{per} \mathrm{kg} / \mathrm{m}^{2}$ increase of BMI. The majority of women in our cohort had a normal BMI 20.0-24.9 ( $\mathrm{n}=111)$, less were categorised as being underweight (BMI <20.0, $\mathrm{n}=34)$ and very few were obese $(\mathrm{BMI}>30.0, \mathrm{n}=9)$. In contrast to previous literature, the mean number of oocytes collected in the obese group was higher than the underweight group, although not to a significant extent (9.6 vs 8.5). This is further evidence that in the context of SEF, patient demographics are dissimilar to the standard IVF population. Obese infertile women may have associated subfertility related to their body habitus, such as those with polycystic ovarian syndrome; however overweight fertile women may not have a discernible reduction in oocyte yield. This is exemplified by previous studies where obese women contributed to $8.9 \%$ of the total cohort, compared to only $2.9 \%$ in our own cohort. ${ }^{24}$ This reinforces further that much of the evidence on prediction of oocyte yield deduced from IVF cycles in infertile women may not be extrapolatable to SEF populations.

At the time of writing $9.7 \%$ of the women who underwent SEF had returned to use their oocytes, a mean of 3.7 years after cryopreservation. A previous study reporting outcomes from 1382 women who underwent SEF at a mean age of 37.7 years showed that $8.7 \%(n=120)$ women returned to use their oocytes after a short follow up time of 2.2 years. ${ }^{25}$ Another study, using a smaller sample of 254 women in Sweden, reported that $15 \%(\mathrm{n}=38)$ returned to use their oocytes after a longer follow up period, returning after a mean of 4 years, ${ }^{26}$ whilst another survey reported just $6 \%$ of 96 women returned to use their oocytes, 2.8 years after undergoing SEF. ${ }^{27}$ Current utilisation rates appear low as some women, particularly those less than 35 years of age may subsequently spontaneously conceive whilst their oocytes are cryopreserved.

The clinical pregnancy rate demonstrated in our cohort of women was $31.7 \%$ with a median age of 43 years old. This is comparable to the average pregnancy rates reported by the Human Fertilisation and Embryology Authority (HFEA) in women aged between $35-37$ years. ${ }^{28}$ Such findings epitomises the reproductive potential of SEF and provides further clinical correlation of the concept. Just under a third (30.5\%) of women who returned to use their stored oocytes attained a livebirth. This is favourable compared to a recent study analysing 10 years of data from two UK clinics who had a success rate, pertaining to livebirths or ongoing pregnancies of $17.5 \% .{ }^{29}$ In that study, which had a similar mean age at cryopreservation of 37 years, successful outcomes including a livebirth or ongoing pregnancy were achieved in $20-23 \%$ of women aged $<40$. In the data presented herein, the success rate in those who cryopreserved oocytes $<40$ years was higher, at $37 \%$ $(\mathrm{n}=11 / 30)$. In the Swedish study that published outcomes of 38 women after SEF, the cumulative live birth rate was $63 \%, 26 \%$ and $0 \%$ in women of ages 36-37, 38-39 and [?]40 years at vitrification respectively. ${ }^{26}$ When stratifying using similar age groups in the cohort presented herein, this data presents findings, with livebirth rates in women who underwent SEF [?]35 years of $33 \%(\mathrm{n}=6), 37.5 \%$ aged $36-39(\mathrm{n}=24)$, and $0 \%$ [?]40 years $(\mathrm{n}=11)$.

In those women who underwent SEF over the age of 40 years old, no women who returned to thaw their oocytes had a successful outcome, and of those, almost three quarters $(72.7 \%)$ had exhausted their oocyte store. This proportion is more than double that reported following assessment of outcomes from the aforementioned Swedish study, where in the 11 women who underwent SEF over the age of 40, none achieved a livebirth, and four (36\%) had exhausted their oocyte supply. ${ }^{26}$ The previously discussed UK study across two centres fared slightly better, with $7 \%$ of those aged 40-42 having a successful outcome. ${ }^{29}$ Whilst the overall numbers remain small, it is clear undergoing SEF aged 40 or over is unlikely to be successful. Not only are the success rates low, but the risk of miscarriage remains elevated, exemplified by the fact the two 
women who suffered miscarriages in this study were both aged $>40$. This is reinforced by data that has identified the risk of miscarriage in women aged $40-44$ is $51 \%{ }^{30}$

This reaffirms that if women choose to undergo SEF, it is likely to offer most benefit to do so by the age of 36. However, caution should be taken advising women to cryopreserve 'too' early. Each case should be individually considered, with appropriate contemplation of the physiological, obstetric, legal and economic considerations. ${ }^{31}$ Although storing oocytes earlier optimises oocyte quality and enhances success rates, the likelihood of spontaneous conception in those additional reproductive years is greater, which would potentially have resulted in unnecessary physical risk and substantial financial burden, in addition to the expense of longer oocyte storage. Moreover, in the context of UK legislation, current HFEA regulations permit the storage of oocytes for a maximum period of 10 years. ${ }^{32}$ Therefore, storing oocytes earlier to optimise future success may be counterproductive, necessitating the need to prematurely use a sperm donor, to prevent the oocytes being discarded. However, following concern regarding the appropriateness of this legal time frame ${ }^{4,33}$ it is possible this legal chronological restriction may be alleviated in the future.

The findings of this study add substantially to published literature to identify factors that impact success following SEF. The associated enhanced ability to counsel women regarding their probability of oocyte yield, and subsequent livebirth is important as some women may not wish to continue with cryopreservation if there is a suboptimal chance of success. Whilst speculative, if women in this cohort were aware they had a very low chance of success, they may have undergone further cycles to optimise their future chances, or indeed if are 40 and above, not embark upon treatment in the first place. Other studies have shown that women are willing to undergo two or more cycles in order to retrieve sufficient numbers of oocytes for storage. ${ }^{34}$ Therefore, it is essential women have the most accurate individualised estimate of their likelihood of future success, in order to make informed choices regarding number of cycles and facilitate the management of future expectations. However, it is paramount that no matter how high the predicted percentage chance of achieving a livebirth, women are made aware that the likelihood of a livebirth cannot be guaranteed. This is important given evidence has suggested women who overestimate expectancy of livebirth, or have few oocytes to preserve often regret freezing, resulting in subsequent emotional and psychological sequelue. ${ }^{35}$

\section{Limitations}

Limitations of this study include that all participants were from a single centre, and as such, there is potential for bias. Moreover, the decision to give either GnRH antagonist or dual trigger were made on an individual basis and based on clinical judgment, including consideration of previous response to stimulation along with baseline AMH and AFC and E2 levels on the day of trigger. The lack of strict criteria introduces the potential for bias in this analysis. All of the above could be minimised by the use of a multi-centre randomised powered study.

\section{Conclusion}

This study highlights that age, BMI, E2, and number of follicles [?]12mm at trigger can predict oocyte yield in SEF cycles. As such, individualising SEF cases with consideration of these markers, should be undertaken to optimise oocyte yield. AMH and AFC are however not independently significantly associated with oocyte yield. Additionally, this is the first study to establish an incidence of OHSS in SEF cycles and establish a significant relationship between age, total AFC, number of follicles [?]12mm and FSH as predictors of OHSS. Moreover, the reproductive outcomes presented highlight that undertaking SEF over the age of 40 years is suboptimal and associated with a low chance of success. Whilst there was no significant difference in livebirth rates in women [?]35 years and aged 36-39, it is advisable that SEF should be undertaken at or prior to the age 36 to optimise outcomes. This data has significant implications for clinical practice, and can be used to individualise care, enhance counselling and manage expectations in women undergoing SEF.

\section{Disclosure of interests}

The authors of this manuscript have no conflicts of interest to declare.

\section{Contribution of authorship}


LSK and BPJ instigated and wrote the manuscript. CH, RO and JG were involved in the data collection. AP carried out the statistical analysis. SS and PS were involved in the critique of the manuscript. JBN was involved in the conception and final critique of the manuscript. All authors reviewed the final draft.

\section{Ethics Approval}

Ethics approval was not necessary for this retrospective study as vitrification of oocytes is a validated technique that should no longer be considered experimental.

\section{Funding}

No funding was required for the purpose of this research or manuscript.

\section{References}

(1) Crawford S, Boulet SL, Kawwass JF, Jamieson DJ, Kissin DM. Cryopreserved oocyte versus fresh oocyte assisted reproductive technology cycles, United States, 2013. Fertil Steril 2017;107(1):110-118.

(2) Jones BP, Saso S, Mania A, Smith JR, Serhal P, Ben Nagi J. The dawn of a new ice age: social egg freezing. Acta Obstet Gynecol Scand 2018;97(6):641-647.

(3) Jones BP, Serhal P, Ben-Nagi J. Social egg freezing should be offered to single women approaching their late thirties: FOR: Women should not suffer involuntary childlessness because they have not yet found a partner. BJOG: An International Journal of Obstetrics \& Gynaecology 2018;125(12):1579-1579.

(4) Jones BP, Kasaven L, L'Heveder A, Jalmbrant M, Green J, Makki M, et al. Perceptions, outcomes, and regret following social egg freezing in the UK; a cross-sectional survey. Acta Obstet Gynecol Scand . 2020;99(3):324-332. doi:10.1111/aogs.13763

(5) Nicoletti C, Tanturri ML. Differences in delaying motherhood across European countries: Empirical evidence from the ECHP. European Journal of Population/Revue europeenne de Demographie 2008;24(2):157183.

(6) Martinelli L, Busatta L, Galvagni L, Piciocchi C. Social egg freezing: a reproductive chance or smoke and mirrors? Croat Med J 2015 Aug;56(4):387-391.

(7) Cobo A, Garrido N, Pellicer A, Remohi J. Six years' experience in ovum donation using vitrified oocytes: report of cumulative outcomes, impact of storage time, and development of a predictive model for oocyte survival rate. Fertil Steril 2015;104(6):1426-1434. e8.

(8) Devesa M, Tur R, Rodriguez I, Coroleu B, Martinez F, Polyzos NP. Cumulative live birth rates and number of oocytes retrieved in women of advanced age. A single centre analysis including 4500 women[?] 38 years old. Human Reproduction 2018;33(11):2010-2017.

(9) Al-Azemi M, Killick SR, Duffy S, Pye C, Refaat B, Hill N, et al. Multi-marker assessment of ovarian reserve predicts oocyte yield after ovulation induction. Human reproduction 2010;26(2):414-422.

(10) Fedorcsak P, Dale PO, Storeng R, Ertzeid G, Bjercke S, Oldereid N, et al. Impact of overweight and underweight on assisted reproduction treatment. Human Reproduction 2004;19(11):2523-2528.

(11) Nelson SM, Klein BM, Arce J. Comparison of antimullerian hormone levels and antral follicle count as predictor of ovarian response to controlled ovarian stimulation in good-prognosis patients at individual fertility clinics in two multicenter trials. Fertil Steril 2015;103(4):923-930. e1.

(12) Vaughan DA, Harrity C, Sills ES, Mocanu EV. Serum estradiol: oocyte ratio as a predictor of reproductive outcome: an analysis of data from> 9000 IVF cycles in the Republic of Ireland. J Assist Reprod Genet 2016;33(4):481-488.

(13) El-Shawarby SA, Khalaf Y. Age-specific serum FSH concentrations and their correlation with the outcome of ovarian stimulation for IVF. Reproductive biomedicine online 2009;18(6):750-755. 
(14) Wirleitner B, Okhowat J, Vištejnová L, Králíčková M, Karlíková M, Vanderzwalmen P, et al. Relationship between follicular volume and oocyte competence, blastocyst development and live-birth rate: optimal follicle size for oocyte retrieval. Ultrasound in Obstetrics \& Gynecology 2018;51(1):118-125.

(15) Ben-Nagi J, Wells D, Doye K, Loutradi K, Exeter H, Drew E, et al. Karyomapping: a single centre's experience from application of methodology to ongoing pregnancy and live-birth rates. Reproductive BioMedicine Online 2017;35(3):264-271.

(16) Jenkins J, Drakeley A, Mathur R. The Management of Ovarian Hyperstimulation Syndrome.RCOG Green Top Guideline no.5, 2006.Reconfirmed 2011.

(17) Majumder K, Gelbaya TA, Laing I, Nardo LG. The use of anti-Mullerian hormone and antral follicle count to predict the potential of oocytes and embryos. European Journal of Obstetrics \& Gynecology and Reproductive Biology 2010;150(2):166-170.

(18) Moreau J, Gatimel N, Simon C, Cohade C, Lesourd F, Parinaud J, et al. Age-specific anti-Mullerian hormone (AMH) levels poorly affects cumulative live birth rate after intra-uterine insemination. European Journal of Obstetrics \& Gynecology and Reproductive Biology: X 2019;3:100043.

(19) Broer S, Dolleman M, Opmeer B, Fauser B, Mol B, Broekmans F. AMH and AFC as predictors of excessive response in controlled ovarian hyperstimulation: a meta-analysis. Hum Reprod Update 2010;17(1):46-54.

(20) Meczekalski B, Czyzyk A, Kunicki M, Podfigurna-Stopa A, Plociennik L, Jakiel G, et al. Fertility in women of late reproductive age: the role of serum anti-Mullerian hormone (AMH) levels in its assessment. J Endocrinol Invest 2016;39(11):1259-1265.

(21) Barad D, Weghofer A, Goyal A, Gleicher N. Age specific anti-mullerian hormone (AMH) levels discriminate at each age between poorer and better oocyte yields. Fertil Steril 2009;92(3):S101.

(22) Wittemer C, Ohl J, Bailly M, Bettahar-Lebugle K, Nisand I. Does body mass index of infertile women have an impact on IVF procedure and outcome? J Assist Reprod Genet 2000;17(10):547-552.

(23) Zhang JJ, Feret M, Chang L, Yang M, Merhi Z. Obesity adversely impacts the number and maturity of oocytes in conventional IVF not in minimal stimulation IVF. Gynecological Endocrinology 2015;31(5):409413.

(24) Christensen MW, Ingerslev HJ, Degn B, Kesmodel US. Effect of female body mass index on oocyte quantity in fertility treatments (IVF): treatment cycle number is a possible effect modifier. A register-based cohort study. PloS one 2016;11(9):e0163393.

(25) Cobo A, Garcia-Velasco JA, Coello A, Domingo J, Pellicer A, Remohi J. Oocyte vitrification as an efficient option for elective fertility preservation. Fertil Steril 2016;105(3):755-764. e8.

(26) Wennberg A, Schildauer K, Brannstrom M. Elective oocyte freezing for non-medical reasons; a six-year report on utilization and IVF results from a Swedish center. Acta obstetricia et gynecologica Scandinavica. 2019 Nov;98(11):1429-34.

(27) Hammarberg K, Kirkman M, Pritchard N, Hickey M, Peate M, McBain J, et al. Reproductive experiences of women who cryopreserved oocytes for non-medical reasons. Human Reproduction 2017;32(3):575581.

(28) Fertilisation H, Authority E. Fertility Treatment 2014-2015 Trends and figures. HFEA Available at https://www.hfea.gov.uk/media/2563/hfea-fertility-trends-and-figures-2017-v2.pdf Accessed 2018;14(08):2018.

(29) Gurtin Z. Unscrambling HFEA data on egg freezing: where are the missing frozen eggs. BioNews 2018:943. 
(30) Andersen AN, Wohlfahrt J, Christens P, Olsen J, Melbye M. Maternal age and fetal loss: population based register linkage study. BMJ 2000;320(7251):1708-1712.

(31) Jones BP, Serhal P, Ben Nagi J. Social egg freezing: Early is not always best. Acta Obstet Gynecol Scand 2018;97(12):1531-1531.

(32) Fertilisation H, Authority E. Fertility treatment in 2013: trends and figures. HFEA, London (Available at http://www.hfea.gov.uk/docs/HFEA_Fertility_treatment_ Trends_and_figures_2014.pdf Last accessed: 8 May 2017) 2014.

(33) Bowen-Simpkins P, Wang JJ, Ahuja KK. The UK' s anomalous 10-year limit on oocyte storage: time to change the law. Reproductive biomedicine online 2018;37(4):387-389.

(34) Nekkebroeck J, Stoop D, Devroey P. O-036 A preliminary profile of women opting for oocyte cryopreservation for non-medical reasons. Human Reproduction 2010;25(20100600).

(35) Greenwood EA, Pasch LA, Hastie J, Cedars MI, Huddleston HG. To freeze or not to freeze: decision regret and satisfaction following elective oocyte cryopreservation. Fertil Steril 2018;109(6):1097-1104. e1.

Table 1 Demographic data and controlled ovarian stimulation variables

\begin{tabular}{ll}
\hline & Median (range) \\
\hline Age (years) & $38.3(26-47)$ \\
BMI $\left(\mathbf{K g} / \mathbf{m}^{2}\right)$ & $22.5(17.1-36.6)$ \\
FSH $(\mathbf{I U} / \mathbf{l})$ & $7.2(1.1-13.7)$ \\
AMH (pmol/l) & $9.9(0.17-56.6)$ \\
Baseline antral follicle count & $12(2-49)$ \\
Number of days of stimulation & $9(4-14)$ \\
Total dose of stimulation IU & $3562.5(700-13875)$ \\
Antral follicles > 12mm on day of trigger & $9(1-50)$ \\
Oestradiol levels on day of trigger (per 1000 pmol/l) & $7.01(0.25-30.26)$ \\
Number of oocytes retrieved & $8(0-37)$ \\
Number of metaphase II & $6(0-28)$ \\
Number of Metaphase I & $0(0-8)$ \\
Number of Germinal Vesicles & $0(0-19)$ \\
\hline
\end{tabular}

\begin{tabular}{llll}
\hline & IRR & $95 \%$ CI & p-value \\
\hline Age $($ years) & 0.92 & $0.91-0.93$ & $<0.001$ \\
BMI $\left(\mathrm{kg} / \mathrm{m}^{2}\right)$ & 0.99 & $0.97-0.99$ & 0.019 \\
AFC & 1.39 & $1.03-1.04$ & $<0.001$ \\
AMH $(\mathrm{pmol} / \mathrm{l})$ & 1.02 & $1.03-1.02$ & $<0.001$ \\
Oestradiol on day of trigger (per 1000 pmol/l) & 1.08 & $1.07-1.09$ & $<0.001$ \\
Number of follicles $>12 \mathrm{~mm}$ on day of trigger & 1.04 & $1.04-1.04$ & $<0.001$ \\
\hline
\end{tabular}

Table 2 The incidence rate ratio (IRR), confidence interval (CI) and p-values to assess the effect of each variable on oocyte yield (univariate analysis)

Table 3 The incidence rate ratio (IRR), confidence interval (CI) and p-values to assess the effect of each variable on oocyte yield (multivariate analysis) 


\begin{tabular}{llll}
\hline & IRR & $95 \%$ CI & p-value \\
\hline & & & \\
\hline Age (years) & IRR & $95 \%$ CI & p-value \\
BMI $\left(\mathrm{kg} / \mathrm{m}^{2}\right)$ & 0.96 & $0.94-0.98$ & $<0.001$ \\
AFC & 1.02 & $1.00-1.03$ & 0.04 \\
AMH $(\mathrm{pmol} / \mathrm{l})$ & 1.00 & $0.99-1.02$ & 0.43 \\
Oestradiol on day of trigger (per 1000 pmol/l) & 1.00 & $0.99-1.01$ & 0.71 \\
Number of follicles $>12 \mathrm{~mm}$ on day of trigger & 1.02 & $1.04-1.07$ & $<0.001$ \\
\hline
\end{tabular}

Table 4 Mann Whitney U, interquartile range (IQR) and p-values to assess the effect of each variable on oocyte yield on OHSS and Non-OHSS groups

\begin{tabular}{llll}
\hline Ovarian reserve marker & OHSS median (IQR) & Non OHSS median (IQR) & p value \\
\hline BMI (Kg/m ${ }^{\mathbf{2}}$ ) & $20.8(5.75)$ & $21.8(4.0)$ & 0.347 \\
Age (years) & $37.0(3.5)$ & $38.0(4.0)$ & $\mathbf{0 . 0 1 9}$ \\
Total AFC & $16.5(22.0)$ & $12.0(8.0)$ & $\mathbf{0 . 0 2 7}$ \\
AMH (pmol/l) & $25.8(25.5)$ & $9.6(10.9)$ & 0.055 \\
No follicles $>\mathbf{1 2 m m}$ & $15.0(11.0)$ & $9.0(7.0)$ & $\mathbf{0 . 0 0 1}^{*}$ \\
FSH (IU/l) & $6.1(2.5)$ & $7.3(3.9)$ & $\mathbf{0 . 0 0 6}^{*}$ \\
Oestradiol (per 1000 pmol/l) & $123.0(154.5)$ & $147.8(137.0)$ & 0.361 \\
\hline
\end{tabular}

\section{Hosted file}

Figure 1* No thaw cycles per age group.pdf available at https://authorea.com/users/364849/ articles/485719-analysis-of-ten-years-of-social-oocyte-cryopreservation-a-researcharticle 